\title{
The host galaxy/AGN connection in nearby early-type galaxies $\star, \star \star$
}

\section{Sample selection and hosts brightness profiles}

\author{
A. Capetti ${ }^{1}$ and B. Balmaverde ${ }^{2}$ \\ 1 INAF - Osservatorio Astronomico di Torino, Strada Osservatorio 20, 10025 Pino Torinese, Italy \\ e-mail: capetti@to.astro.it \\ 2 Universitá di Torino, Via Giuria 1, 10125 Torino, Italy \\ e-mail: balmaverde@ph. unito.it
}

Received 22 March 2005 / Accepted 27 April 2005

\begin{abstract}
This is the first of a series of three papers exploring the connection between the multiwavelength properties of AGNs in nearby early-type galaxies and the characteristics of their hosts. We selected two samples, both with high resolution $5 \mathrm{GHz}$ VLA observations available and providing measurements down to $1 \mathrm{mJy}$ level, reaching radio-luminosities as low as $10^{19} \mathrm{~W} \mathrm{~Hz}^{-1}$. We focus on the 116 radio-detected galaxies as to boost the fraction of AGN with respect to a purely optically selected sample. Here we present the analysis of the optical brightness profiles based on archival HST images, available for 65 objects. We separate early-type galaxies on the basis of the slope of their nuclear brightness profiles, into core and power-law galaxies following the Nuker's scheme, rather than on the traditional morphological classification (i.e. into E and S0 galaxies). Our sample of AGN candidates is indistinguishable, when their brightness profiles are concerned, from galaxies of similar optical luminosity but hosting weaker (or no) radio-sources. We confirm previous findings that relatively bright radiosources $\left(L_{\mathrm{r}}>10^{21.5} \mathrm{~W} \mathrm{~Hz}^{-1}\right)$ are uniquely associated to core galaxies. However, below this threshold in radio-luminosity core and power-law galaxies coexist and they do not show any apparent difference in their radio-properties. Not surprisingly, since our sample is deliberately biased to favour the inclusion of active galaxies, we found a higher fraction of optically nucleated galaxies. Addressing the multiwavelength properties of these nuclei will be the aim of the two forthcoming papers.
\end{abstract}

Key words. galaxies: active - galaxies: bulges - galaxies: elliptical and lenticular, cD - galaxies: nuclei - galaxies: structure

\section{Introduction}

It is becoming increasingly clear that most (if not all) galaxies host a supermassive black hole (SMBH) in their centers (e.g. Kormendy \& Richstone 1995). The tight relationships between the SMBH mass and the stellar velocity dispersion (Ferrarese \& Merritt 2000; Gebhardt et al. 2000) as well as with the mass of the spheroidal component of their host galaxies (e.g. Marconi \& Hunt 2003) clearly indicate that they follow a common evolutionary path. As recently demonstrated by Heckman et al. (2004) the synchronous growth of SMBH (traced by energy output of AGNs) and galaxies (evidenced by their star formation rate) is at work even now, in the nearby Universe. But despite this fundamental breakthrough in our understanding of the

^ Based on observations obtained at the Space Telescope Science Institute, which is operated by the Association of Universities for Research in Astronomy, Incorporated, under NASA contract NAS 5-26555.

$\star \star$ Figures 1-3, 6 and 7 are only available in electronic form at http://www. edpsciences.org
SMBH/galaxy system and the fact that historically the first evidences for the existence of SMBH were provided by the nuclear activity in form of an Active Galactic Nucleus (AGN), we still lack a clear picture of the precise relationship between AGN and host galaxies. For example, while spiral galaxies preferentially harbour radio-quiet AGN, early-type galaxies host both radio-loud and radio-quiet AGN. Similarly, radio-loud AGN are generally associated with the most massive SMBH as there is a median shift between the radio-quiet and radio-loud distribution, but both distributions are broad and overlap considerably (e.g. Dunlop et al. 2003).

In the last decade, thanks to HST imaging, a new picture of the properties of nearby galaxies also emerged. Nearly all galaxies have singular starlight distributions with surface brightness diverging as $\Sigma(r) \sim r^{-\gamma}$, with $\gamma>0$ (e.g. Lauer et al. 1995) and the distribution of cusp slopes in both stellar luminosity density and projected surface brightness is bimodal (Gebhardt et al. 1996; Faber et al. 1997). In some cases $\gamma$ decreases only slowly toward the center and a steep $\gamma>0.5$ cusp continues to the HST resolution limit; these systems are 
classified as "power-law" galaxies. In other objects the projected profile breaks to a shallow inner cusp with $\gamma \leq 0.3$ and these form the class of "core galaxies". A small number of "intermediate" galaxies have been also identified (e.g. Ravindranath et al. 2001) that have cusp slopes with $0.3<\gamma<$ 0.5 , but the ensemble of cusp slopes of the spheroidal component of galaxies is still bimodal. Faber et al. examined how the central structure correlates with other galaxy properties, showing that luminous early-type galaxies preferentially have cores, whereas most fainter spheroids have power-law profiles. Moreover, cores are slowly rotating and have boxy isophotes, while power-laws rotate rapidly and are disky. This scheme fits nicely with the revision of the Hubble sequence proposed by Kormendy \& Bender (1996).

Clearly, these recent developments provide us with a new framework in which to explore the connection between host galaxies and AGNs. In particular, early-type galaxies appear to be the critical class of objects, in which core and power-law galaxies coexist, i.e. where the transition between the two profiles classes occur.

This study must be limited to samples of relatively nearby galaxies. In fact the Nuker classification can only be obtained when the nuclear region, potentially associated to a shallow cusp, can be well resolved. The radius at which the break in the brightness profiles occurs are in the range $10 \mathrm{pc}-1 \mathrm{kpc}$; the most compact cores will be barely resolved already at a distance of $40 \mathrm{Mpc}$ (where $10 \mathrm{pc}$ subtend an angle of $0{ }^{\prime} 05$ ) even in the HST images. Furthermore, high quality radio-images are required for an initial selection of AGN candidates. As discussed in more details in Sect. 2 two such large samples of nearby early-type galaxies have been already studied at radiowavelengths and they will represent the starting point for our analysis.

In order to explore the multiwaveleghts properties of these candidates AGN we will take advantage on the substantial coverage supplied by both the HST and Chandra archives when nearby galaxies are concerned. The high resolution and sensitivity of these instruments will enable us to isolate the nuclear emission, if present, in both the optical and X-ray band down to unprecedented low luminosity levels. This will open the possibility of building three-bands diagnostic diagrams comparing the radio, optical and X-ray emission, to identify genuine low luminosity AGN and to explore their spectral energy distributions.

The plan of this series of papers is as follows: in this first part we will define the samples to be examined, collect the available HST images, analyze the galaxies brightness profiles and compare our results with other samples studied in the past. In the two forthcoming papers we will firstly discuss the properties of objects with a core profile, while power-law galaxies will be discussed and compared in a third paper.

We adopt a Hubble constant of $H_{0}=75 \mathrm{~km} \mathrm{~s}^{-1} \mathrm{Mpc}^{-1}$.

\section{Sample selection and description}

For our purposes we must study a large sample of nearby objects for which radio observations combining relatively high resolution, high frequency and sensitivity are available, in order to minimize the contribution from radio-emission not related to the galaxy's nucleus and confusion from background sources.

Two studies in the literature fulfill these requirements. Wrobel \& Heeschen (1991) and Sadler et al. (1989) presented $5 \mathrm{GHz}$ VLA radio-images of two samples of nearby early-type galaxies with a resolution between $3^{\prime \prime}$ and $5^{\prime \prime}$ and a flux limit of $\sim 1 \mathrm{mJy}$. The two samples were selected with a very similar strategy. More specifically, Wrobel (1991) extracted a northern sample of galaxies from the CfA redshift survey (Huchra et al. 1983) satisfying the following criteria: (1) $\delta_{1950} \geq 0$, (2) photometric magnitude $B \leq 14$; (3) heliocentric velocity $\leq$ $3000 \mathrm{~km} \mathrm{~s}^{-1}$, and (4) morphological Hubble type $T \leq-1$, for a total number of 216 galaxies. Sadler et al. (1989) selected a southern sample of E and S0 galaxies brighter than magnitude 14 , with $\delta \leq-32$, (the Parkes sample). They subsequently selected a subsample of 116 galaxies with the further restriction of $\delta \geq-45$ to be observable with the VLA. The only substantial difference between the two samples is that Sadler et al. did not impose a distance limit. Nonetheless, the threshold in optical magnitude effectively limits the sample to a recession velocity of $\sim 6000 \mathrm{~km} \mathrm{~s}^{-1}$. Consequently their sample has a median recession velocity larger by a factor of $\sim 2$ higher than for the Wrobel sample and it contains galaxies which are optically (and radio) brighter by an average factor of 4 .

Here we focus on the radio-detected sources of both samples, building a radio-flux limited sample of AGN candidates. Having excluded three galaxies from the Local Group (Leo I and II, Ursa Minor), Wrobel \& Heeschen detected 67 galaxies, while 49 were detected by Sadler et al. (1989). In the HST public archive we found images taken with WFPC2, NICMOS or ACS, for a total of 65 objects, 48 and 17 from the Wrobel sample (hereafter Sample I) and from the Sadler et al. sample (hereafter Sample II) respectively. These galaxies form our final sample.

In Tables 1 and 2 we provide the basic data for the selected galaxies namely the recession velocity (corrected for infall of the Local Group towards Virgo from the LEDA database), the $K$ band magnitude from the Two Micron All Sky Survey (2MASS) (available for all but one source), the $V$ band galactic extinction, the total radio- luminosity at $5 \mathrm{GHz}$, the radio-core flux - when this can be isolated in the VLA maps.

\section{HST data and surface brightness profiles}

HST images of the selected galaxies were taken with a variety of instruments and filters. When multiple datasets are available for a given object, we give preference to those obtained with ACS or WFPC2 in the $F 814 W$ or $F 555 W$ filters to improve the uniformity of the images used. However, when significant dust structures are present in the optical, we used the infrared NICMOS images if present in the archive. All data were retrieved from the public HST archive and were calibrated by the standard On The Fly Re-processing (OTFR) system. In Tables 3 and 4 we give the instrument/filter combination for the images used to study the brightness profile which are presented in Figs. 1 through 4.

Several objects show peculiar morphologies on the HST scale which prevent any attempt to produce a fit to their 
Table 1. Galaxies in sample I: (1) UGC name; (2) alternative optical identifications; (3) recession velocity in $\mathrm{km} \mathrm{s}^{-1}$; (4) total $K$ band galaxy's magnitude from 2MASS; (5) galactic extinction in the $V$ band from NED; (6) total $5 \mathrm{GHz}$ radio-flux [mJy] from ${ }^{a}$ Wrobel \& Heeschen (1991) or ${ }^{b}$ Wrobel (1991); (7) $5 \mathrm{GHz}$ radio-core [mJy].

\begin{tabular}{|c|c|c|c|c|c|c|}
\hline Name & Alt. Name & $V$ & $m_{K}$ & Gal. $A_{\mathrm{V}}$ & $F_{\text {radio }}$ & $F_{\text {core }}$ \\
\hline UGC 0167 & NGC 0063 & 1197 & $9.257 \pm 0.027$ & 0.369 & $3.6 \pm 0.2^{a}$ & \\
\hline UGC 0968 & NGC 0524 & 2429 & $7.163 \pm 0.012$ & 0.274 & $1.4 \pm 0.1^{a}$ & \\
\hline UGC 5292 & NGC 3032 & 1652 & $9.648 \pm 0.024$ & 0.056 & $3.7 \pm 0.7^{a}$ & \\
\hline UGC 5617 & NGC 3226 & 1410 & $8.570 \pm 0.037$ & 0.075 & $3.6 \pm 0.2^{a}$ & \\
\hline UGC 5663 & NGC 3245 & 1456 & $7.862 \pm 0.010$ & 0.083 & $3.3 \pm 0.2^{a}$ & \\
\hline UGC 5902 & NGC 3379, M 105 & 951 & $6.270 \pm 0.018$ & 0.081 & $0.7 \pm 0.1^{a}$ & \\
\hline UGC 5959 & NGC 3414, Arp 162 & 1651 & $7.981 \pm 0.014$ & 0.081 & $5.0 \pm 0.2^{a}$ & \\
\hline UGC 6153 & NGC 3516 & 2902 & $8.512 \pm 0.027$ & 0.140 & $15.5 \pm 1.7^{a}$ & \\
\hline UGC 6297 & NGC 3607 & 1022 & $7.276 \pm 0.021$ & 0.069 & $2.6 \pm 0.1^{a}$ & \\
\hline UGC 6605 & NGC 3773 , Mrk 743 & 1052 & $10.696 \pm 0.063$ & 0.089 & $1.6 \pm 0.1^{a}$ & \\
\hline UGC 6742 & NGC 3870, Mrk 186 & 981 & $10.820 \pm 0.042$ & 0.052 & $4.4 \pm 0.9^{a}$ & \\
\hline UGC 6834 & NGC 3928, Mrk 190 & 1207 & $9.674 \pm 0.025$ & 0.065 & $7.6 \pm 1.0^{a}$ & \\
\hline UGC 6860 & NGC 3945 & 1500 & $7.526 \pm 0.025$ & 0.094 & $1.0 \pm 0.1^{a}$ & \\
\hline UGC 6877 & IC 0745, Mrk 1308 & 1162 & - & 0.069 & $0.8 \pm 0.1^{a}$ & \\
\hline UGC 6946 & NGC 3988 & 1285 & $7.365 \pm 0.010$ & 0.053 & $83 \pm 2^{b}$ & \\
\hline UGC 6985 & NGC 4026 & 1173 & $7.584 \pm 0.019$ & 0.073 & $1.4 \pm 0.1^{a}$ & \\
\hline UGC 7005 & NGC 4036 & 1690 & $7.562 \pm 0.019$ & 0.078 & $2.9 \pm 0.2^{a}$ & \\
\hline UGC 7103 & NGC 4111 & 1006 & $7.553 \pm 0.018$ & 0.048 & $2.3 \pm 0.1^{a}$ & \\
\hline UGC 7142 & NGC 4143 & 1135 & $7.853 \pm 0.009$ & 0.042 & $6.7 \pm 0.3^{a}$ & \\
\hline UGC 7203 & NGC 4168 & 2392 & $8.437 \pm 0.021$ & 0.120 & $4.5 \pm 0.2^{a}$ & \\
\hline UGC 7256 & NGC 4203 & 1263 & $7.406 \pm 0.014$ & 0.040 & $12.5 \pm 0.4^{a}$ & \\
\hline UGC 7311 & NGC 4233 & 2417 & $8.784 \pm 0.032$ & 0.079 & $1.9 \pm 0.1^{a}$ & \\
\hline UGC 7329 & NGC 4250 & 2308 & $9.140 \pm 0.027$ & 0.069 & $6.4 \pm 1.0^{a}$ & \\
\hline UGC 7360 & NGC 4261, 3C 270 & 2238 & $7.263 \pm 0.028$ & 0.060 & $8300 \pm 400^{b}$ & 315 \\
\hline UGC 7386 & NGC 4278 & 804 & $7.184 \pm 0.011$ & 0.095 & $351 \pm 11^{b}$ & \\
\hline UGC 7494 & NGC 4374, M 84 & 1004 & $6.222 \pm 0.023$ & 0.134 & $2800 \pm 100^{b}$ & 350 \\
\hline UGC 7515 & NGC 4385, Mrk 052 & 2172 & $9.776 \pm 0.046$ & 0.082 & $3.7 \pm 0.2^{a}$ & \\
\hline UGC 7575 & NGC 4435 & 887 & $7.297 \pm 0.016$ & 0.100 & $1.2 \pm 0.1^{a}$ & \\
\hline UGC 7614 & NGC 4459 & 1294 & $7.152 \pm 0.011$ & 0.153 & $0.8 \pm 0.1^{a}$ & \\
\hline UGC 7629 & NGC 4472, M 49 & 940 & $5.396 \pm 0.025$ & 0.074 & $95 \pm 10^{b}$ & 57 \\
\hline UGC 7654 & NGC 4486, M 87 & 1361 & $5.812 \pm 0.019$ & 0.074 & $71900 \pm 400^{b}$ & 4000 \\
\hline UGC 7718 & NGC 4526 & 665 & $6.473 \pm 0.020$ & 0.074 & $3.1 \pm 0.2^{a}$ & \\
\hline UGC 7760 & NGC 4552, M 89 & 392 & $6.728 \pm 0.024$ & 0.136 & $121 \pm 4^{b}$ & \\
\hline UGC 7797 & NGC 4589 & 2233 & $7.758 \pm 0.023$ & 0.093 & $21.0 \pm 0.7^{a}$ & \\
\hline UGC 7878 & NGC 4636 & 1112 & $6.422 \pm 0.035$ & 0.092 & $45.0 \pm 9.0^{b}$ & 6 \\
\hline UGC 7898 & NGC 4649, M 60 & 1231 & $5.739 \pm 0.021$ & 0.088 & $24.0 \pm 2.0^{b}$ & 18 \\
\hline UGC 8355 & IC 875 & 3051 & $10.296 \pm 0.027$ & 0.036 & $0.5 \pm 0.1^{a}$ & \\
\hline UGC 8499 & NGC 5198 & 2778 & $8.896 \pm 0.023$ & 0.077 & $1.5 \pm 0.1^{a}$ & \\
\hline UGC 8675 & NGC 5273 & 1316 & $8.665 \pm 0.024$ & 0.033 & $1.2 \pm 0.1^{a}$ & \\
\hline UGC 8745 & NGC 5322 & 2096 & $7.160 \pm 0.027$ & 0.045 & $20 \pm 1^{b}$ & 14 \\
\hline UGC 9655 & NGC 5813 & 2025 & $7.413 \pm 0.031$ & 0.182 & $2.1 \pm 0.1^{a}$ & \\
\hline UGC 9692 & NGC 5838 & 1450 & $7.581 \pm 0.019$ & 0.176 & $2.0 \pm 0.1^{a}$ & \\
\hline UGC 9706 & NGC 5846 & 1896 & $6.949 \pm 0.026$ & 0.182 & $5.3 \pm 0.3^{a}$ & \\
\hline UGC 9723 & NGC 5866 & 1034 & $6.873 \pm 0.018$ & 0.044 & $7.4 \pm 0.3^{a}$ & \\
\hline UGC 10656 & NGC 6278 & 2981 & $8.994 \pm 0.017$ & 0.204 & $1.2 \pm 0.1^{a}$ & \\
\hline UGC 12317 & NGC 7465, Mrk 313 & 2046 & $9.542 \pm 0.021$ & 0.260 & $3.7 \pm 0.2^{a}$ & \\
\hline UGC 12329 & NGC 7468, Mrk 314 & 2164 & $11.575 \pm 0.066$ & 0.282 & $1.4 \pm 0.1^{a}$ & \\
\hline UGC 12759 & NGC 7743 & 1725 & $8.418 \pm 0.028$ & 0.233 & $2.7 \pm 0.1^{a}$ & \\
\hline
\end{tabular}


Table 2. Galaxies in sample II: (1) optical name; (2) alternative optical identifications; (3) recession velocity in $\mathrm{km} \mathrm{s}^{-1}$; (4) total $K$ band galaxy's magnitude from 2MASS; (5) galactic extinction in the $V$ band; (6) total $5 \mathrm{GHz}$ [mJy] radio-flux from ${ }^{a}$ Sadler et al. (1989) or ${ }^{b}$ Slee et al. (1994); (7) radio-core flux [mJy].

\begin{tabular}{llrcrrr}
\hline \hline Name & Alt. Name & $V$ & \multicolumn{1}{c}{$m_{K}$} & Gal. $A_{\mathrm{V}}$ & $F_{\text {radio }}$ & $F_{\text {core }}$ \\
\hline NGC 1316 & Fornax A & 1548 & $5.587 \pm 0.019$ & 0.069 & $65800^{b}$ & 26 \\
NGC 1380 & E358-G28 & 1621 & $6.869 \pm 0.022$ & 0.058 & $1.7^{a}$ & \\
NGC 1399 & E358-G45 & 1217 & $6.306 \pm 0.027$ & 0.043 & $342^{b}$ & 10 \\
NGC 2328 & E309-G16 & 913 & $9.931 \pm 0.042$ & 0.295 & $2.5^{a}$ & \\
NGC 3258 & E375-G37 & 2598 & $8.306 \pm 0.024$ & 0.279 & $52^{b}$ & 3.7 \\
NGC 3268 & E375-G45 & 2600 & $8.153 \pm 0.029$ & 0.341 & $22.8^{a}$ & \\
NGC 3557 & E377-G16 & 2857 & $7.203 \pm 0.017$ & 0.327 & $299^{b}$ & 10 \\
NGC 3706 & E378-G06 & 2827 & $7.902 \pm 0.019$ & 0.306 & $19^{b}$ & 7.6 \\
NGC 4373 & E322-G06 & 3221 & $7.651 \pm 0.022$ & 0.265 & $12.7^{a}$ & \\
NGC 4696 & E322-G91 & 2767 & $7.142 \pm 0.024$ & 0.376 & $1393^{b}$ & 55 \\
NGC 5128 & Cen A & 390 & $3.942 \pm 0.016$ & 0.381 & $126000^{b}$ & 6984 \\
NGC 5419 & E384-G39 & 4044 & $7.518 \pm 0.032$ & 0.239 & $390^{b}$ & 15 \\
NGC 6958 & E341-G15 & 2566 & $8.400 \pm 0.018$ & 0.149 & $18.1^{a}$ & \\
IC 1459 & E406-G30 & 1498 & $6.805 \pm 0.025$ & 0.053 & $1016^{b}$ & \\
IC 3370 & E322-G14 & 2773 & $7.858 \pm 0.021$ & 0.309 & $6.4^{a}$ & \\
IC 4296 & E383-G39 & 3613 & $7.502 \pm 0.021$ & 0.204 & $1604^{b}$ & 210 \\
IC 4931 & E339-G23 & 5820 & $8.662 \pm 0.030$ & 0.234 & $0.9^{a}$ & \\
\hline
\end{tabular}

surface brightness. These include spiral-like objects, objects with a clumpy morphology suggestive of star forming regions, and galaxies with severe dust obscuration. In three cases this is probably due to the fact that the only HST observations available were taken in the ultraviolet with the $F 300 W$ filter. All galaxies discarded at this stage, $10+1$ in sample I and II respectively, are marked with a dagger in Tables 3 and 4. It is interesting to note that all 11 complex galaxies have $M_{K}>-22.5$ (with the exception of UGC 7329 with $M_{K}=-23.0$ ), substantially fainter than the median of the regular galaxies $\left(M_{K}=\right.$ $-24.5)$.

When available we take the parameters describing the Nuker law fit from the literature. This is the case for $21+3$ objects in the two sub-samples. For the remaining 30 objects we used IRAF task "ellipse" to fit elliptical isophotes to the galaxies (Jedrzejewski 1987). These include a few objects for which the classification was based on pre-refurbishment HST data from Byun et al. (1996) ${ }^{1}$.

Prominent dust features are often present and they are masked before fitting; in the radial range corresponding to dust structures, we usually preferred to fix the isophotes center, position angle and ellipticity to the values of the last isophote not affected by dust, in order to stabilize the fit. When the dust feature cover a large azimuthal range (e.g. in the case of circumnuclear disks) no masking can be used and the resulting profiles will be necessarily affected. Similarly we masked all background stars or other spurious sources present in the field of view. The background was measured at the edges of images

\footnotetext{
${ }^{1}$ During the preparation of this paper Lauer et al. (2004) published fit to the HST images of additional 7 objects of our sample. The classification in core and power-law coincide with those given in the Tables 3 and 4 with the only exception of NGC 3706: while they conclude that this is a core galaxy, we adopt a more conservative description as a complex profile.
}

and subtracted. A few objects fill the whole available field of view; in these cases we treated the background level as an extra free parameter in the Nuker fitting. The background level is uncorrelated with all other parameters except for the large scale slope $\beta$ and it can be easily measured and accounted for, particularly in these well exposed images. The resulting onedimensional major-axis (background subtracted) profiles are presented in Figs. 5 through 7. Errors in the surface brightness are not be displayed as they rarely exceed the $1 \%$ level.

On these profiles we performed a fit with a Nuker law in the form

$I(r)=I_{\mathrm{b}} 2^{(\beta-\gamma) / \alpha}\left(\frac{r_{\mathrm{b}}}{r}\right)^{\gamma}\left[1+\left(\frac{r}{r_{\mathrm{b}}}\right)^{\alpha}\right]^{(\gamma-\beta) / \alpha}$.

The parameter $\beta$ measures the slope of the outer region of the brightness profile, $r_{\mathrm{b}}$ is the break radius (corresponding to a brightness $I_{\mathrm{b}}$ ) where the profile flattens to a smaller slope measured by the parameter $\gamma . \alpha$ sets the sharpness of the transition between the inner and outer profile. All models were convolved with the appropriate one dimensional Point Spread Function profile before they are compared to the data.

We minimized the value of the $\chi^{2}$, starting from a first guess eye fit and then exploring the value of $\chi^{2}$ over a uniform grid in the 5-dimensional parameter space. The grid was then refined in the region close to the $\chi^{2}$ minimum to reach an accuracy on each parameter of 0.01 and finally a downhill simplex minimization algorithm was applied to convergence. As the behaviour at the smaller radii might be affected by the details of the Point Spread Function used for the convolution of the models (such as PSF dependence of chip location, telescope breathing and so on) we preferred to exclude points with $r \leq 0$.' 1 from the fit. In several objects the HST images show the presence of a prominent nuclear point source. In these cases we flagged the innermost points of the profile to an extent which depends on 

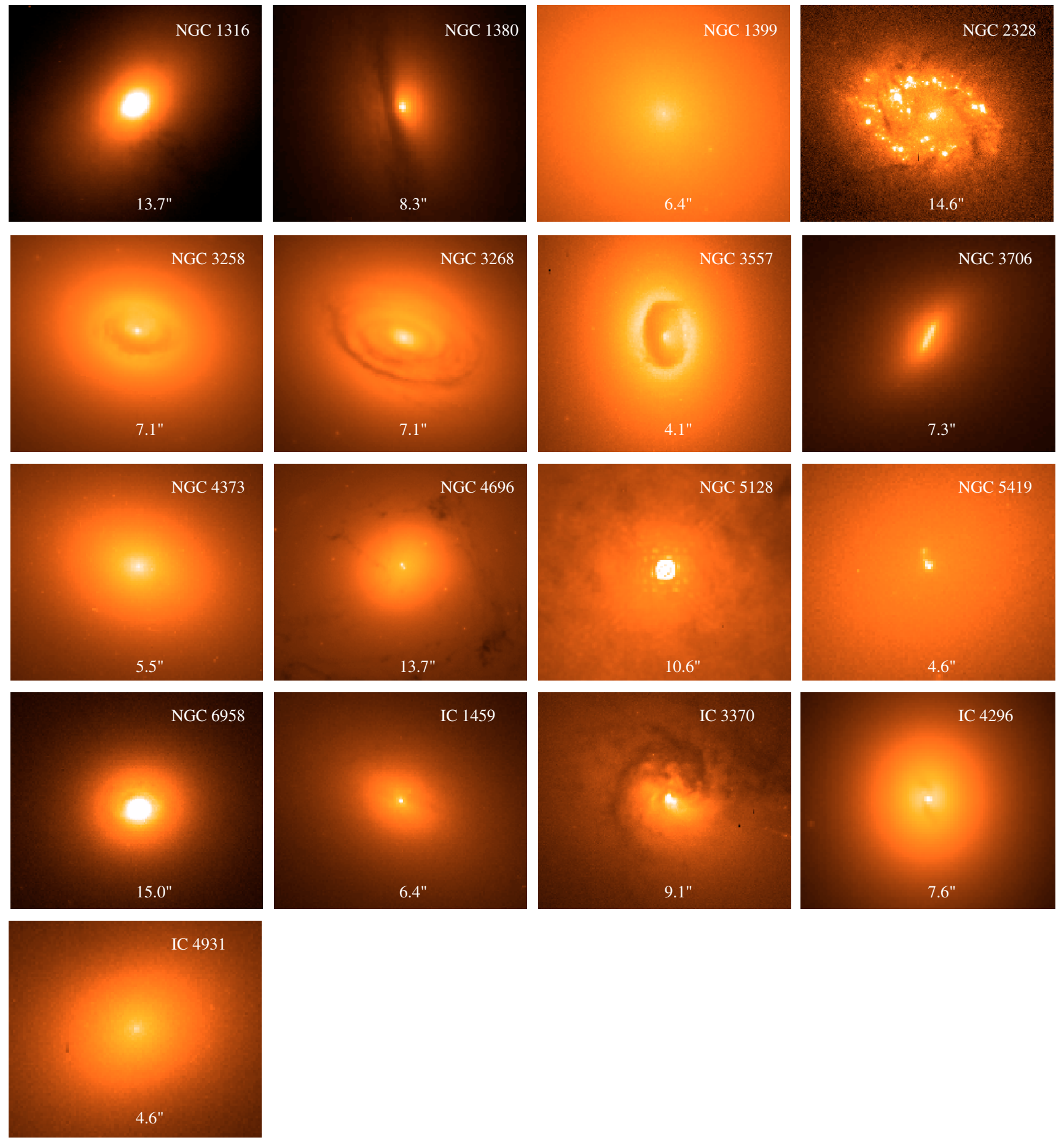

Fig. 4. HST images of the galaxies of the sample.

the point source intensity, usually the radius where the brightness profile shows the characteristic up-turn.

In Figs. 5 through 7 we superposed the best fit with a Nuker law to the actual data in the top panel, while the lower panel presents the residuals. Regions masked in the fitting procedure have their residuals marked with a cross instead of a circle.

Three galaxies show brightness profiles that cannot be reproduced by a Nuker law. These are marked in Tables 3 and 4 as "complex profiles". This leaves us with 51 objects. In some cases, a good fit can only be obtained by flagging a range of radii; in most cases this is due to a depression in the brightness profiles that can be easily associated to dusty regions in the HST images. In other cases, localized region of excess are found over an otherwise well defined Nuker profile. Clearly there is some level of arbitrariness in separating complex profiles from those only slightly disturbed. To reflect this issue we mark with an "?" all sources (6) in which we flagged a significant portion of the brightness profile. However, in 

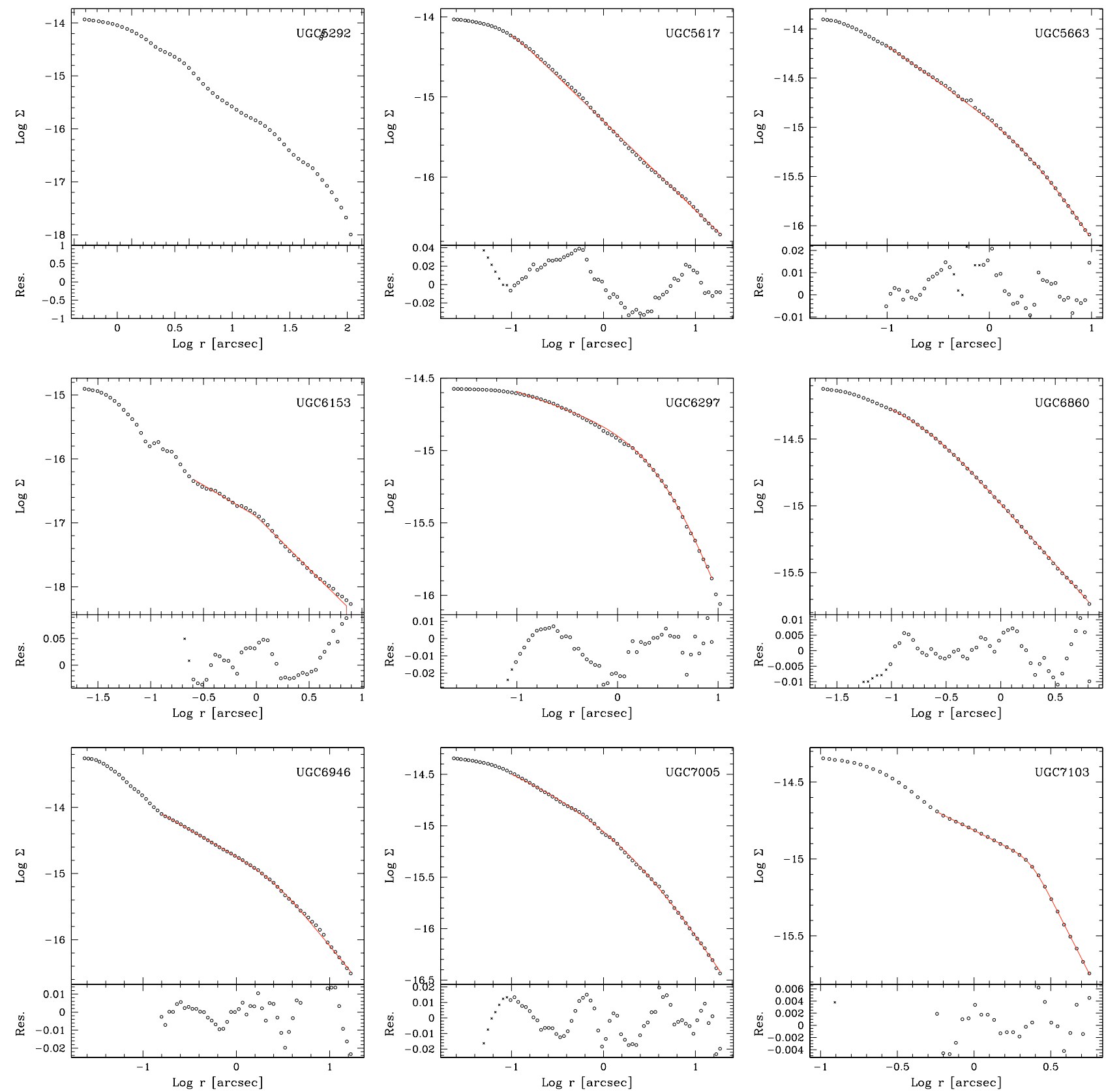

Fig. 5. Surface brightness (in unit of $\mathrm{erg} \mathrm{s}^{-1} \mathrm{~cm}^{-2} \AA^{-1}$ ) profiles of all galaxies lacking a core/power-law classification in the literature. Spiral-like or complex sources are excluded. The solid line represents the best fit for all objects satisfactorily reproduced with a Nuker law. Residuals are presented in the bottom panel. Points excluded from the fit have their residuals marked with a cross instead of a circle.

general, residuals show large scale fluctuations with amplitude of $\leq 1-2 \%$ over the whole profile, with only two exceptions reaching locally values of $5 \%$. This implies that overall a Nuker law reproduces quite well the galaxies profiles.

The parameters derived from the Nuker fits are reported in Tables 3 and 4 . The brightness at the break radius $\mu_{\mathrm{b}}$ has been converted to the standard Cousin-Johnson system and corrected for Galactic extinction. Break radii are in arcseconds.

Sources are separated into power-law and core galaxies adopting the standard values, i.e. $\gamma \leq 0.3$ are classified as core-galaxies, while $\gamma \geq 0.5$ are power-law galaxies. We also considered as power-law galaxies, following the scheme of
Lauer et al. (1995), all those objects (6) in which no break in the brightness profile is seen at the HST resolution limit. More specifically, we included in this class the galaxies with $r_{\mathrm{b}} \leq 0 ! 2$, as the region below the break is not sufficiently sampled (when not simply unresolved) to provide an accurate estimate of $\gamma$. Potentially, these objects might be core galaxies located at a sufficiently large distance so that a compact shallow core cannot be resolved. Indeed, there is one core-galaxy in our sample, UGC 7760 (NGC 4552, M 89) with $v=392 \mathrm{~km} \mathrm{~s}^{-1}$, with a well resolved core, $r_{\mathrm{b}}=0$. ' 49 corresponding to $13 \mathrm{pc}$, which would have been misclassified with this scheme if it was located at a larger distance. Thus we prefer in the remaining 
Table 3. Nuker parameters for sample I.

\begin{tabular}{|c|c|c|c|c|c|c|c|c|}
\hline Name & Image & $\alpha$ & $\beta$ & $\gamma$ & $r_{\mathrm{b}}$ & $\mu_{\mathrm{b}}$ & Ref. & Classification \\
\hline UGC 0167 & WFPC2/F300W & \multicolumn{5}{|c|}{$\dagger$ Complex } & (1) & - \\
\hline UGC 0968 & NICMOS/F160W & 0.68 & 1.69 & 0.03 & 1.41 & 13.73 & (2) & Core \\
\hline UGC 5292 & NICMOS/F160W & \multicolumn{5}{|c|}{ Complex profile } & (1) & - \\
\hline UGC 5617 & WFPC2/F702W & - & 1.11 & - & $<0.20$ & $<15.17$ & (1) & Power-law* \\
\hline UGC 5663 & WFPC $2 / F 702 W$ & 1.87 & 1.59 & 0.73 & 2.69 & 16.48 & (1) & Power-law \\
\hline UGC 5902 & NICMOS/F160W & 1.82 & 1.45 & 0.18 & 1.58 & 12.80 & (2) & Core \\
\hline UGC 5959 & WFPC2/F702W & 1.40 & 1.45 & 0.83 & 1.72 & 16.46 & (3) & Power-law \\
\hline UGC 6153 & NICMOS/F160W & 42.60 & 1.64 & 0.97 & 1.61 & 13.97 & (1) & Power-law? \\
\hline UGC 6297 & WFPC2/F814W & 1.51 & 1.97 & 0.23 & 3.22 & 15.86 & (1) & Core \\
\hline UGC 6605 & WFPC $2 / F 300 W$ & \multicolumn{5}{|c|}{$\dagger$ Complex } & (1) & - \\
\hline UGC 6742 & WFPC $2 / F 814 W$ & \multicolumn{5}{|c|}{$†$ Complex } & (1) & - \\
\hline UGC 6834 & WFPC $2 / F 606 W$ & \multicolumn{5}{|c|}{$\dagger$ Spiral } & (1) & - \\
\hline UGC 6860 & WFPC $2 / F 814 W$ & - & 0.92 & - & $<0.20$ & $<14.57$ & (1) & Power-law* \\
\hline UGC 6877 & NICMOS/F160W & \multicolumn{5}{|c|}{$†$ Complex } & (1) & - \\
\hline UGC 6946 & WFPC2/F547M & 2.94 & 1.75 & 0.80 & 2.61 & 16.76 & (1) & Power-law \\
\hline UGC 6985 & NICMOS/F160W & 0.88 & 1.50 & 0.68 & 0.84 & 12.40 & (2) & Power-law \\
\hline UGC 7005 & WFPC2/F547M & 0.89 & 1.46 & 0.36 & 1.98 & 17.08 & (1) & Interm. \\
\hline UGC 7103 & NICMOS/F160W & 10.50 & 1.97 & 0.50 & 2.35 & 12.90 & (1) & Power-law \\
\hline UGC 7142 & NICMOS/F160W & 1.26 & 2.18 & 0.59 & 3.11 & 14.26 & (2) & Power-law \\
\hline UGC 7203 & WFPC2/F702W & 1.43 & 1.39 & 0.17 & 2.02 & 17.45 & (3) & Core \\
\hline UGC 7256 & WFPC $2 / F 814 W$ & 2.13 & 1.41 & 0.62 & 1.50 & 15.33 & (1) & Power-law \\
\hline UGC 7311 & WFPC $2 / F 702 W$ & 2.49 & 1.95 & 0.64 & 3.43 & 18.11 & (1) & Power-law? \\
\hline UGC 7329 & WFPC $2 / F 300 W$ & \multicolumn{5}{|c|}{$\dagger$ Complex } & (1) & - \\
\hline UGC 7360 & NICMOS/F160W & 2.38 & 1.43 & 0.00 & 1.62 & 13.58 & (2) & Core \\
\hline UGC 7386 & NICMOS/F160W & 1.63 & 1.39 & 0.02 & 0.97 & 12.80 & (2) & Core \\
\hline UGC 7494 & NICMOS/F160W & 2.15 & 1.50 & 0.13 & 2.39 & 13.35 & (2) & Core \\
\hline UGC 7515 & WFPC2/F606W & \multicolumn{5}{|c|}{$†$ Complex } & (1) & - \\
\hline UGC 7575 & WFPC $2 / F 814 W$ & 1.25 & 2.13 & 0.33 & 3.45 & 16.22 & (1) & Interm. \\
\hline UGC 7614 & WFPC2/F814W & 6.51 & 1.12 & 0.66 & 0.47 & 13.97 & (1) & Power-law \\
\hline UGC 7629 & NICMOS/F160W & 1.89 & 1.29 & 0.04 & 2.63 & 13.52 & (2) & Core \\
\hline UGC 7654 & NICMOS/F160W & 2.56 & 2.78 & 0.28 & 9.41 & 15.51 & (1) & Core \\
\hline UGC 7718 & WFPC2/F814W & \multicolumn{5}{|c|}{$\dagger$ Dusty } & (1) & - \\
\hline UGC 7760 & WFPC2/F555W & 3.03 & 1.06 & 0.00 & 0.49 & 15.24 & (4) & Core \\
\hline UGC 7797 & NICMOS/F160W & 1.09 & 1.18 & 0.11 & 0.21 & 12.05 & (2) & Core \\
\hline UGC 7878 & NICMOS/F160W & 1.69 & 1.56 & 0.13 & 3.44 & 14.60 & (2) & Core \\
\hline UGC 7898 & WFPC2/F555W & 2.44 & 1.27 & 0.19 & 3.83 & 16.95 & (1) & Core \\
\hline UGC 8355 & WFPC $2 / F 702 W$ & 0.85 & 1.81 & 1.07 & 1.87 & 17.65 & (3) & Power-law \\
\hline UGC 8499 & WFPC2/F702W & 2.61 & 1.13 & 0.23 & 0.16 & 14.79 & (3) & Power-law* \\
\hline UGC 8675 & NICMOS/F160W & 7.03 & 1.32 & 0.37 & 0.65 & 13.96 & (2) & Interm. \\
\hline UGC 8745 & WFPC2/F814W & 1.11 & 1.45 & 0.00 & 0.68 & 14.59 & (4) & Core \\
\hline UGC 9655 & WFPC2/F702W & 1.90 & 1.33 & 0.00 & 0.73 & 15.77 & (3) & Core \\
\hline UGC 9692 & NICMOS/F160W & 4.35 & 2.57 & 0.93 & 4.35 & 14.84 & (2) & Power-law \\
\hline UGC 9706 & WFPC2/F702W & 2.62 & 1.07 & 0.00 & 1.52 & 17.12 & (1) & Core \\
\hline UGC 9723 & NICMOS/F160W & 2.50 & 1.51 & 0.00 & 2.99 & 13.61 & (1) & Core? \\
\hline UGC 10656 & WFPC2/F702W & 0.76 & 1.62 & 0.55 & 0.60 & 15.69 & (3) & Power-law \\
\hline UGC 12317 & WFPC $2 / F 606 W$ & \multicolumn{5}{|c|}{$\dagger$ Complex } & (1) & - \\
\hline UGC 12329 & WFPC $2 / F 814 W$ & \multicolumn{5}{|c|}{$\dagger$ Complex } & (1) & - \\
\hline UGC 12759 & NICMOS/F160W & 5.36 & 1.38 & 0.50 & 0.16 & 11.59 & (2) & Power-law* \\
\hline
\end{tabular}

References: (1) this work; (2) Ravindranath et al. (2001); (3) Rest et al. (2001); (4) Carollo et al. (1997).

* Power-law galaxies with $r_{\mathrm{b}} \leq 0.2 ;$ ? tentative classification. 
Table 4. Nuker parameters for sample II.

\begin{tabular}{|c|c|c|c|c|c|c|c|c|}
\hline Name & Image & $\alpha$ & $\beta$ & $\gamma$ & $r_{\mathrm{b}}$ & $\mu_{\mathrm{b}}$ & Ref. & Classification \\
\hline NGC 1316 & NICMOS/F160W & 1.88 & 1.06 & 0.13 & 0.52 & 11.54 & (1) & Core \\
\hline NGC 1380 & NICMOS/F160W & - & 0.81 & - & $<0.20$ & $<12.08$ & (1) & Power-law* \\
\hline NGC 1399 & WFPC2/F814W & 2.04 & 1.21 & 0.10 & 2.19 & 15.37 & (1) & Core \\
\hline NGC 2328 & WFPC2/F814W & \multicolumn{5}{|c|}{$\dagger$ Spiral } & (1) & - \\
\hline NGC 3258 & $\mathrm{ACS} / F 814 W$ & 2.10 & 1.51 & 0.00 & 1.15 & 15.59 & (1) & Core? \\
\hline NGC 3268 & $\mathrm{ACS} / F 814 W$ & 2.49 & 1.64 & 0.13 & 1.51 & 15.87 & (1) & Core \\
\hline NGC 3557 & WFPC $2 / F 555 W$ & 2.62 & 1.44 & 0.00 & 1.60 & 16.38 & (1) & Core? \\
\hline NGC 3706 & WFPC $2 / F 555 W$ & \multicolumn{5}{|c|}{ Complex profile } & (1) & - \\
\hline NGC 4373 & WFPC $2 / F 814 W$ & 1.69 & 1.45 & 0.08 & 1.19 & 15.25 & (1) & Core \\
\hline NGC 4696 & WFPC2/F814W & 6.63 & 0.86 & 0.10 & 1.40 & 16.36 & (5) & Core \\
\hline NGC 5128 & NICMOS/F222M & 1.68 & 1.30 & 0.10 & 2.56 & 11.98 & (1) & Core \\
\hline NGC 5419 & WFPC2/F555W & 2.01 & 1.35 & 0.05 & 2.11 & 17.56 & (1) & Core \\
\hline NGC 6958 & WFPC $2 / F 814 W$ & - & 1.22 & - & $<0.20$ & $<13.63$ & (1) & Power-law*? \\
\hline IC 1459 & WFPC $2 / F 814 W$ & 0.81 & 2.04 & 0.02 & 1.82 & 15.15 & (4) & Core \\
\hline IC 3370 & WFPC $2 / F 555 W$ & \multicolumn{5}{|c|}{ Complex profile } & (1) & - \\
\hline IC 4296 & NICMOS/F160W & 2.14 & 1.90 & 0.00 & 1.44 & 13.89 & (1) & Core \\
\hline IC 4931 & WFPC $2 / F 814 W$ & 2.08 & 1.46 & 0.09 & 0.99 & 15.74 & (5) & Core \\
\hline
\end{tabular}

References: (1) this work; (4) Carollo et al. (1997); (5) Laine et al. (2003).

* Power-law galaxies with $r_{\mathrm{b}} \leq 0.2$; ? tentative classification.

of the paper to mark this class differently from objects with well established power-law profiles. In the sample there also 3 galaxies (two among those analyzed in this work) with an intermediate value of $\gamma$. See Table 5 for a summary of the breakdown of the objects into the different classes.

Since we were forced to use several filters for the analysis of the surface brightness profile, there is the possibility that the description of a given galaxy changes when observed in different bands. However, the comparison of the parameters derived from Nuker fits on the same galaxy imaged with HST in different filters (Ravindranath et al. 2001) indicates that, although differences are present, the classification into power-law and core galaxies is independent on wavelength. Nonetheless, we prefer to explore this issue in more detail for our sample. The images used in this work range from the $V$ to the $H$ band. We here consider the 21 galaxies for which images taken in both these bands are available, to test the robustness of the Nuker classification over the largest breadth of wavelength. Nine objects must be discarded since their $V$ band images are saturated (3), heavily affected by dust (4) or as the presence of a single image in the archive prevents cosmic-ray rejection (2). Of the 12 remaining objects, brightness profiles of 11 galaxies have been already presented in the literature (see the articles cited in Table 3, Lauer et al. 2004 and Faber et al. 1997), leaving to us only the analysis of the $V$ band image of UGC 7494 (M 84). No discordant Nuker classification has been found, further supporting its independence on the observing band.

As discussed by several authors, a formal estimate of errors on the resulting Nuker parameters can not be performed (e.g. Byun et al. 1996). Since we are mostly interested in separating galaxies with shallow cusps from those with steep cusps, we explored how the variations of $\gamma$ are reflected in the fit and in
Table 5. Nuker classification summary.

\begin{tabular}{lcc}
\hline \hline Class & \# Sample I & \# Sample II \\
\hline Core galaxies & $16+1 ?$ & $10+2 ?$ \\
Power-law galaxies & $10+5^{*}+2 ?$ & $1 *+1 ?$ \\
Interm. galaxies & 3 & 0 \\
Complex profiles & 1 & 2 \\
Complex morphologies & 10 & 1 \\
\hline Total & 48 & 17 \\
\hline
\end{tabular}

? Tentative classification; * power-law galaxies with $r_{\mathrm{b}} \leq 0.2$.

the residuals. We focus on UGC 7575, one of the two galaxies for which we found an intermediate value of $\gamma=0.33$. Its best fit (see Fig. 6) shows residuals always smaller than $\pm 1.5 \%$, quite typical for all fitted galaxies. In Fig. 8 we present the Nuker fit obtained forcing $\gamma$ to 0.43 , a value 0.1 larger with respect to the best fit, leaving all other parameters free to vary. Large and systematic residuals appear toward the center, reaching $5 \%$ at $0{ }^{\prime} 1$. Although this does not provide us with a precise error estimate, it indicates that the error on $\gamma$ are generally significantly smaller than 0.1 , in agreement with the analysis performed by Byun et al. This implies that except for a few border-line objects our classification is robust.

\section{Discussion}

We are now in the position to explore how the multiwavelength properties of the galaxies of our sample are related to the brightness profiles and how they compare with results obtained on purely optically selected samples. In Fig. 9 we plot 


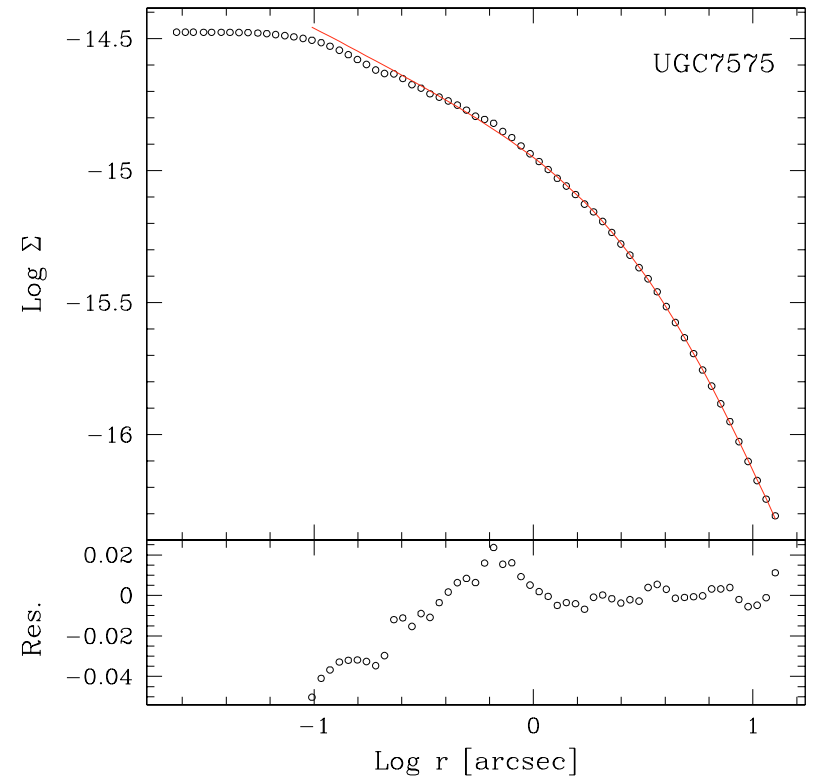

Fig. 8. Nuker fit to UGC 7575 forcing the value of $\gamma$ to be 0.1 larger than the best fit value of 0.33 , while leaving al other parameters free to vary. This representative example is used to illustrate that the error on this parameter is in general smaller than 0.1 .

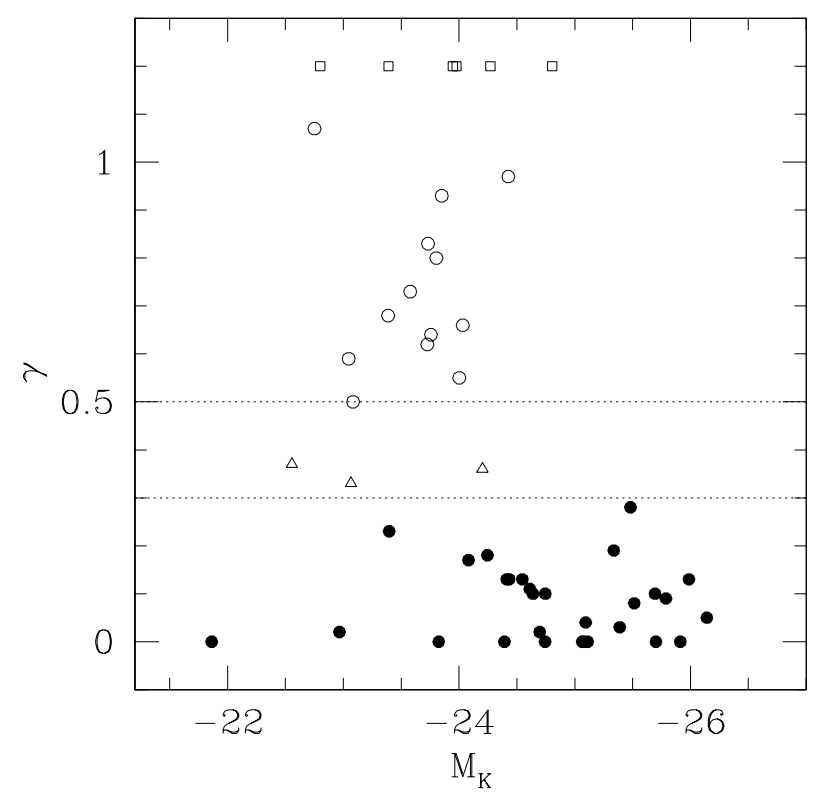

Fig. 9. Logarithmic inner slope derived from the Nuker fit vs. galaxy's $K$ band magnitude. Galaxies belonging to sample I and II are separated on the basis of the value of $\gamma$. Core galaxies have $\gamma \leq 0.3$ (filled circles), intermediate galaxies have $0.3<\gamma<0.5$ (empty triangles), power-law galaxies have $\gamma \geq 0.5$ (empty circles). Power-law galaxies in which no break in the brightness profile is seen above the resolution limit are located arbitrarily at $\gamma=1.2$ and are marked with empty squares.

the absolute $K$ band magnitude $M_{K}$ derived from 2MASS measurements $^{2}$ with the asymptotic slope $\gamma$. We recover the

\footnotetext{
${ }^{2}$ We preferred to use the $K$ band luminosities, despite all previous studies have used optical measurements, since the near-infrared light is a better tracer of the stellar mass and less subject to the effects of
}

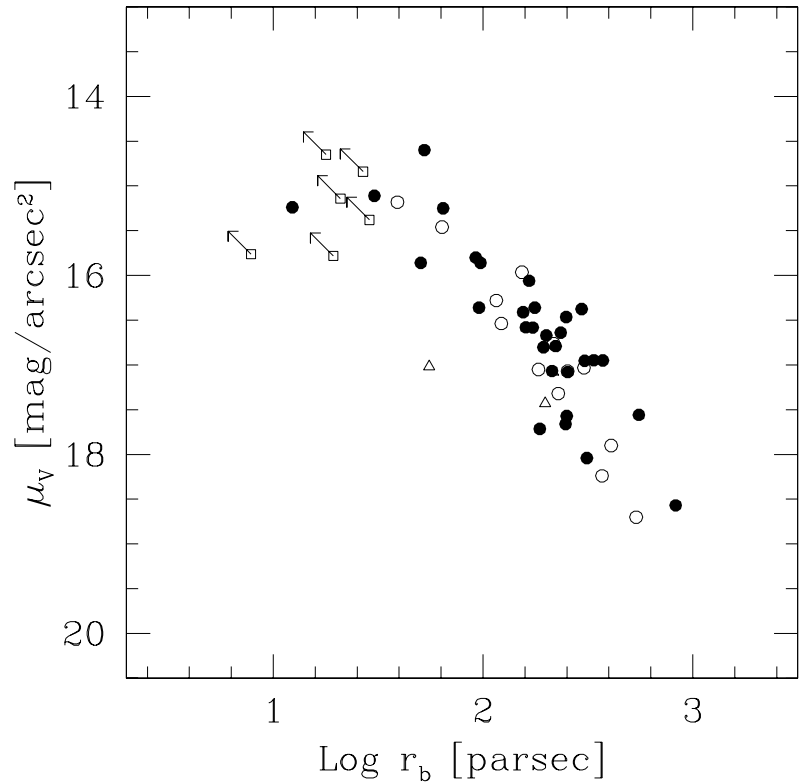

Fig. 10. Surface brightness at the break radius $\mu_{\mathrm{b}}$ vs. break radius $r_{\mathrm{b}}$. Symbols as in Fig. 9, i.e. filled circles for core galaxies, empty circles for power-laws, triangles for intermediate galaxies, squares for galaxies with no break in the brightness profile.

already known difference between core and power-law galaxies in terms of absolute magnitude. The most luminous galaxies are exclusively associated to core profiles. Nonetheless, coregalaxies are observed over a broad range of luminosity extending from $M_{K} \sim-26.5$ down to $M_{K} \sim-21.8$, more than two magnitudes above and below the break in the luminosity function, $M_{K}^{*}=-24.3$ (Huang et al. 2003). Leaving aside the objects in which no break in the brightness profile is observed, the brightest power-law galaxy has $M_{K}=-24.2$. Therefore, there is a substantial overlap between the two classes as far as their luminosity is concerned.

The well defined correlation between break radius and surface brightness at the break, which have been described as a fundamental plane for the galaxies cores, is also recovered (see Fig. 10) ${ }^{3}$. The comparison with previous works (see e.g. Fig. 8 in Faber et al. and the compilation provided by De Ruiter et al. 2005) indicate that the $r_{\mathrm{b}}$ vs. $\mu_{\mathrm{b}}$ relationship we obtain is in very good agreement from that seen in the other samples studied, purely optically selected. We conclude that our sample does not reflect any significant difference in the host galaxies with respect to the overall population of early-type galaxies, despite it has been selected imposing a minimum radio-flux level.

The only significant difference is the higher fraction of nucleated galaxies; this fraction varies considerably in previous studies e.g. from $\sim 15 \%$ in Laine et al. to $\sim 40 \%$ in Lauer et al. Although we defer the analysis of the nuclear prop-

extinction. Furthermore, 2MASS provides us with a uniform and accurate set of measurements. The conversion to e.g. the $V$ band can be obtained using the average color for E galaxies, $V-K=3.3$, see Mannucci et al. (2001).

${ }^{3}$ We converted surface brightness in the different filters into the $V$ band using the average colors for $\mathrm{E} / \mathrm{S} 0$ tabulated by Mannucci et al. (2001). 


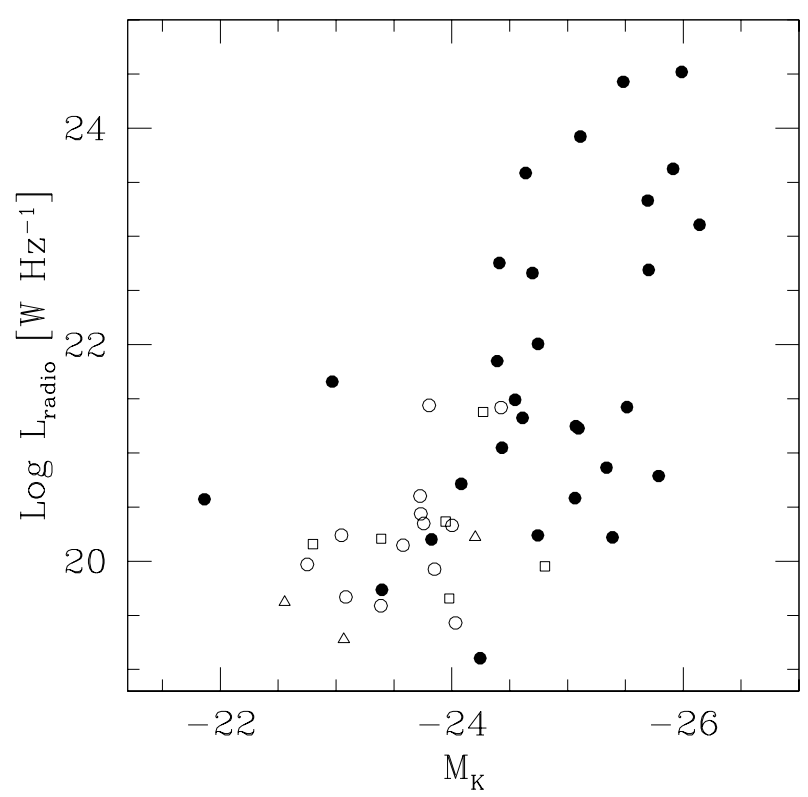

Fig. 11. Host galaxies magnitude vs. radio-luminosity. Symbols: filled circles for core galaxies, empty circles for power-laws, triangles for intermediate galaxies, squares for galaxies with no break in the brightness profile.

erties to the forthcoming papers, we note that in our sample we detected an optical (or infrared) nucleus in $\sim 65 \%$ of the objects. This is not at all unexpected, since our sample is deliberately biased to favour the inclusion of active galaxies.

Concerning the relationship between host's magnitude and radio-power this is visually presented in Fig. 11. Several authors in the past discussed this relationship (e.g. Auriemma et al. 1977) that can be described as a trend for which brighter galaxies have a higher probability to be strong radioemitters with respect to less luminous galaxies. By separating core and power-law galaxies a clearer picture emerges. Only core-galaxies are radio-emitters at level larger than $L_{\mathrm{r}}>$ $2.5 \times 10^{21} \mathrm{~W} \mathrm{~Hz}^{-1}$. This is in line with the result found by De Ruiter et al. (2005); their analysis of the brightness profiles of HST images of radio-galaxies extracted from the B2 sample, as well as from nearby $3 \mathrm{C}$ sources, shows that all host galaxies of these relatively powerful radio-sources have a core profile. Figure 12 provides us with a different view of this same effect, by comparing $\gamma$ with $L_{\mathrm{r}}$. It also shows that, when the two classes are separated, there is no dependence of the radioluminosity on the value of $\gamma$, e.g. smaller values of $\gamma$ in core galaxies are not associated to the brightest radio-sources.

However, below this threshold, the two populations of early-type galaxies cannot be readily differentiated. We are left with the ambiguity on what is the driving mechanism at the origin of this threshold in radio-luminosity for power-law galaxies: it can be due to an intrinsic difference with respect to core galaxies, but it might also be related to the difference in the host galaxy's luminosity, since no strong radio-source $\left(L_{\mathrm{r}}>10^{22} \mathrm{~W} \mathrm{~Hz} \mathrm{~s}^{-1}\right)$ is associated to a host with $M_{K}>-24$, regardless of the profile class.

Considering separately the two classes, the (optically) brightest core galaxies are indeed associated to the strongest

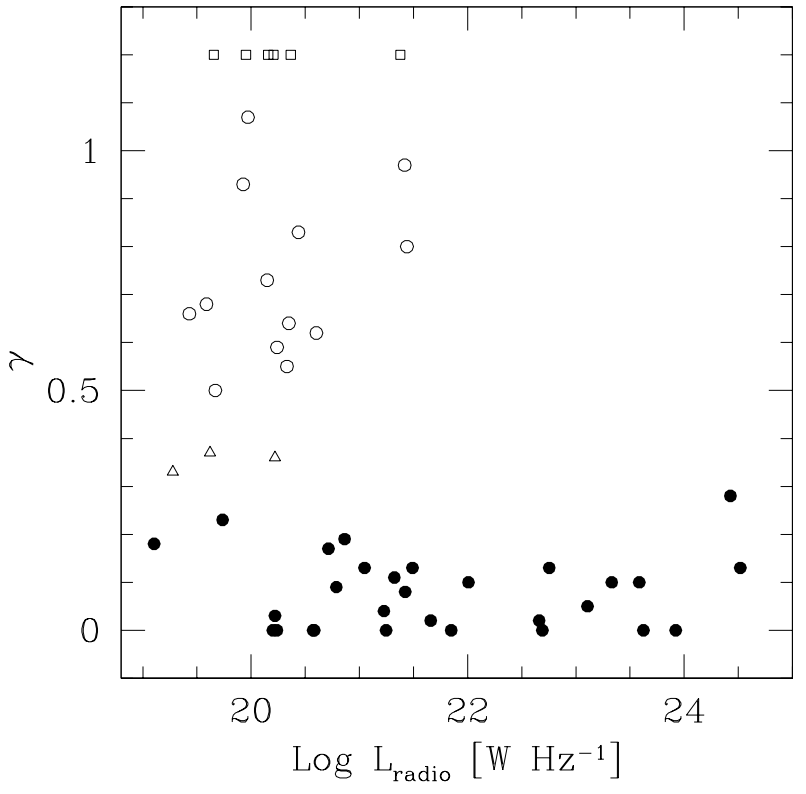

Fig. 12. Logarithmic inner slope vs. radio-luminosity. Symbols: filled circles for core galaxies, empty circles for power-laws, triangles for intermediate galaxies, squares for galaxies with no break in the brightness profile.

radio-sources. However, this effect cannot be simply described as a correlation between $L_{\mathrm{r}}$ and $M_{K}$ since they show a very large spread, covering as much as 3 dex in radio-luminosity at a given magnitude. Furthermore, this trend does not extend to the lowest host magnitudes.

If we now consider the galaxies with intermediate nuclear slope and those in which no break in the brightness profile is seen (marked as triangles and squares respectively in Fig. 11) they are all relatively faint radio-sources, below the threshold for power-law galaxies discussed above. On the other hand, these objects are in the range of relatively low optical luminosity where few radio-bright objects are found.

\section{Summary and conclusions}

Aim of this series of three papers is to explore the connection between the host galaxies and the AGN activity in nearby early-type galaxies. The new developments in our understanding of the relationship between the supermassive black-holes and galaxies properties as well as in the structure of the central regions of nearby galaxies warrant to re-explore this classical issue in a new framework. In particular, early-type galaxies are the critical class of objects. The two classes defined on the basis of their brightness profiles (core and power-law galaxies) coexist in early-type galaxies and they host both radio-quiet and radio-loud AGN.

A robust classification of the brightness profile can only be obtained for nearby objects since the most compact cores $(r \sim 10 \mathrm{pc})$ are barely resolved even in the HST images already at a distance of $40 \mathrm{Mpc}$. We then focus on two nearby samples of early-type galaxies well studied at radio-wavelengths in the past and more specifically on 116 galaxies (out a complete sample of 312 objects) that have been detected above the 
$1 \mathrm{mJy}$ threshold in VLA images at $5 \mathrm{GHz}$ by Wrobel (1991) and Sadler et al. (1989). Imposing a radio-flux limit boosts the fraction of AGN with respect to a purely optically selected sample and, at the same time, provides us with a crucial information (their radio-luminosity) on the nuclear activity of these objects. Due to their proximity and to the low radio-flux limit we will be able to explore the AGN properties in objects with a radio-power as low as $L_{\mathrm{r}} \sim 10^{19} \mathrm{~W} \mathrm{~Hz}^{-1}$ (or, equivalently, to $v L_{\mathrm{r}} \sim 10^{36} \mathrm{erg} \mathrm{s}^{-1}$ ).

In this first paper we analyze the archival HST images for this radio-selected sub-sample. They are available for 65 objects. Having discarded 11 objects with a complex morphology, we fit elliptical isophotes and derive the brightness profile for the remaining galaxies. With only 3 exceptions these profiles are well reproduced by a Nuker law fit. We are then able to separate early-type galaxies on the basis of the slope of their nuclear brightness profiles $(\gamma)$, into core $(\gamma \leq 0.3)$ and power-law ( $\gamma \geq 0.5$ ) galaxies; we also found 3 galaxies with an intermediate slope $(0.3<\gamma<0.5)$. We preferred this classification with respect to the traditional morphological scheme (i.e. E and S0 galaxies) since it is well known that it is often difficult to unambiguously distinguish between these two classes of objects (van den Bergh 2004).

We recovered the already known difference in the luminosity between core and power-law galaxies, since the most luminous galaxies are exclusively core galaxies. Below $M_{K} \sim$ -24.2 , however, the two classes coexist and core galaxies are associated to objects as faint as $M_{K} \sim-21.8$. A well defined correlation between the radius and the surface brightness at the profile break, $r_{\mathrm{b}}$ and $\mu_{\mathrm{b}}$, the "core fundamental plane", is present also in our sample and it is indistinguishable from that seen in the other samples, purely optically selected, studied. Thus, our sample does not reflect any significant difference in the host galaxies with respect to the normal galaxies population.

Concerning the relationship between host's magnitude and radio-power, we found that only core-galaxies are radioemitters at level larger than $L_{\mathrm{r}}>2.5 \times 10^{21} \mathrm{~W} \mathrm{~Hz}^{-1}$, confirming the suggestions by De Ruiter et al. (2005). But below this threshold the two populations of early-type galaxies cannot be readily differentiated. Since no strong radio-source $\left(L_{\mathrm{r}}>10^{22} \mathrm{~W} \mathrm{~Hz}^{-1}\right)$ is associated to a host with $M_{K}>-24$ regardless of the profile class, we are left with the ambiguity on the origin of this threshold in radio-luminosity for power-law galaxies: it can be due both to a different nuclear structure of the two classes or to a difference in the host galaxy's luminosity, since power-law galaxies only scarcely populate the high end of the optical luminosity distributions, where the brightest radio-sources are found.

The most significant difference concerning the optical properties of our sample with respect to previous studies is the higher fraction of nucleated galaxies, since we detected an optical (or infrared) nucleus in $\sim 65 \%$ of the objects. This is not at all unexpected, since our sample is deliberately biased to favour the inclusion of active galaxies. Addressing the multiwavelength properties of these nuclei will be the aim of the two forthcoming papers of the series.
Acknowledgements. This research has made use of the NASA/IPAC Extragalactic Database (NED) (which is operated by the Jet Propulsion Laboratory, California Institute of Technology, under contract with the National Aeronautics and Space Administration), of the NASA/ IPAC Infrared Science Archive (which is operated by the Jet Propulsion Laboratory, California Institute of Technology, under contract with the National Aeronautics and Space Administration) and of the LEDA database.

\section{Appendix A: Notes on the individual sources}

UGC 5292: the presence of a bright point source, combined with a complex large scale profile prevents the fitting of this source.

UGC 5663: despite the presence of extended patches of dust, the masking procedure allows us to derive its brightness profile which is well fitted by a Nuker law.

UGC 6153: dust patches cover a large fraction of the nuclear regions of this galaxy and a bright point source is present. Nonetheless, the profile is relatively well behaved and suggests a classification as power-law. The residuals larger than average for the other sources makes it tentative.

UGC 6297: the brightness profile is well reproduced by a Nuker law, despite the presence of dust filaments.

UGC 7311: the large scale edge-on dusty disk covers most of the galaxy for $r<0$. 7 . However, we derived a much larger break radius $\left(r_{\mathrm{b}}=3.43^{\prime \prime}\right)$ and concluded that the classification is robust.

UGC 7614: the presence of an inclined large scale disk induces relatively large fluctuations in the outer profile, but the relatively unperturbed morphology at small radii allows us a robust classification as a power-law galaxy.

UGC 7575: the situation for this object is similar to that of UGC 7614, with a large scale disk but little dust in the innermost $1^{\prime \prime}$, leading to a clear power-law classification.

UGC 9723: in this object an almost exactly edge-on dusty disk is present, but nonetheless the spheroidal component can be accurately traced, after masking down to $\sim 2^{\prime \prime}$. The break radius derived from the Nuker fit is substantially larger $\sim 3^{\prime \prime}$, suggesting a tentative core classification.

NGC 1316: despite the presence of extended patches of dust, the brightness profile is remarkably smooth and well fitted by a Nuker law.

NGC 1380: after masking the off-center dust lane, the brightness profile is smooth and well fitted by a power-law. No flattening is seen before the resolution limit.

NGC 3258: a circum-nuclear well defined dusty disk prevents the study of the brightness profile for $r<0$.' 9 . The innermost points of the profiles suggest the presence of a flat core. The best fit value is found for $r_{\mathrm{b}}=11^{\prime \prime} 15$, i.e. apparently the profile breaks before the edge of the disk. Forcing the value of $\gamma$ to 0.3 requires a strong monotonic increase of dust obscuration toward the center; at $r<0$.' 1 the surface brightness should be depressed by a factor 2.5 , difficult to reach considering the presence of starlight in front of the disk. These considerations suggest a classification as a likely core galaxy. 
NGC 3268: dust filaments cover the region between 0 '. 25 and 1 '.2. Nonetheless the profile is characteristic of a core galaxy.

NGC 3557: the behaviour galaxy is very similar to NGC 3258, see above. Here the break radius is at $r_{\mathrm{b}}=11^{\prime \prime} 60$, while the dusty disk has a radius of $r=0$ ' $^{\prime} 95$. The brightness deficit at the center in this case for $\gamma=0.3$ would be of a factor 2.2, again suggesting a classification as a likely core galaxy.

NGC 3706: the central region of this galaxy has a peculiar morphology, being extremely elongated. The brightness profile has a steep outer slope, which increases below 0'.4 (approximately the size of the elongated feature) then changing to a flat plateau. We conservatively classify this profile as complex.

NGC 5128: the fit has been performed on a dust-corrected image kindly provided by A. Marconi, obtained combining $H$ and $K$ band HST images of this source. The central $1^{\prime \prime}$ is flagged due to the presence of a strong point source.

NGC 5419: the central $0{ }^{\prime} \prime 15$ are flagged due to the presence of a point source. A fainter second point source (flagged in the fitting) is located close to the nucleus.

NGC 6958: this galaxy shows a well defined power-law decrease with a constant slope $\beta=1.22$, after excluding a rather extended region with a light excess reaching 0.2 dex. No flattening is seen before the resolution limit.

IC 3370: dust patches cover a large fraction of the nuclear regions of this galaxy. The resulting profile is quite complex and no fit has been performed.

IC 4296: the circum-nuclear dusty disk seen in the optical HST images (Laine et al. 2003) affects only marginally the NICMOS image used by us. The central 0!' 4 are flagged due to the presence of a point source.

\section{References}

Auriemma, C., Perola, G. C., Ekers, R. D., et al. 1977, A\&A, 57, 41 Byun, Y.-I., Grillmair, C. J., Faber, S. M., et al. 1996, AJ, 111, 1889

Dunlop, J. S., McLure, R. J., Kukula, M. J., et al. 2003, MNRAS, 340, 1095

Faber, S. M., Tremaine, S., Ajhar, E. A., et al. 1997, AJ, 114, 1771

Ferrarese, L., \& Merritt, D. 2000, ApJ, 539, L9

Gebhardt, K., Bender, R., Bower, G., et al. 2000, ApJ, 539, L13

Gebhardt, K., Richstone, D., Ajhar, E. A., et al. 1996, AJ, 112, 105

Heckman, T. M., Kauffmann, G., Brinchmann, J., et al. 2004, ApJ, 613, 109

Huang, J.-S., Glazebrook, K., Cowie, L. L., \& Tinney, C. 2003, ApJ, 584,203

Huchra, J., Davis, M., Latham, D., \& Tonry, J. 1983, ApJS, 52, 89

Jedrzejewski, R. I. 1987, MNRAS, 226, 747

Kormendy, J., \& Bender, R. 1996, ApJ, 464, L119

Kormendy, J., \& Richstone, D. 1995, ARA\&A, 33, 581

Laine, S., van der Marel, R. P., Rossa, J., et al. 2003, AJ, 126, 2717

Lauer, T. R., Ajhar, E. A., Byun, Y.-I., et al. 1995, AJ, 110, 2622

Lauer, T. R., Faber, S. M., Gebhardt, K., et al. 2004, ArXiv Astrophysics e-prints

Mannucci, F., Basile, F., Poggianti, B. M., et al. 2001, MNRAS, 326, 745

Marconi, A., \& Hunt, L. K. 2003, ApJ, 589, L21

Ravindranath, S., Ho, L. C., Peng, C. Y., Filippenko, A. V., \& Sargent, W. L. W. 2001, AJ, 122, 653

Sadler, E. M., Jenkins, C. R., \& Kotanyi, C. G. 1989, MNRAS, 240, 591

Slee, O. B., Sadler, E. M., Reynolds, J. E., \& Ekers, R. D. 1994, MNRAS, 269, 928

van den Bergh, S. 2004, ApJ, 601, L37

Wrobel, J. M. 1991, AJ, 101, 127

Wrobel, J. M., \& Heeschen, D. S. 1991, AJ, 101, 148 
A. Capetti and B. Balmaverde: The host/AGN connection in nearby galaxies, Online Material p 1

\section{Online Material}


A. Capetti and B. Balmaverde: The host/AGN connection in nearby galaxies, Online Material p 2
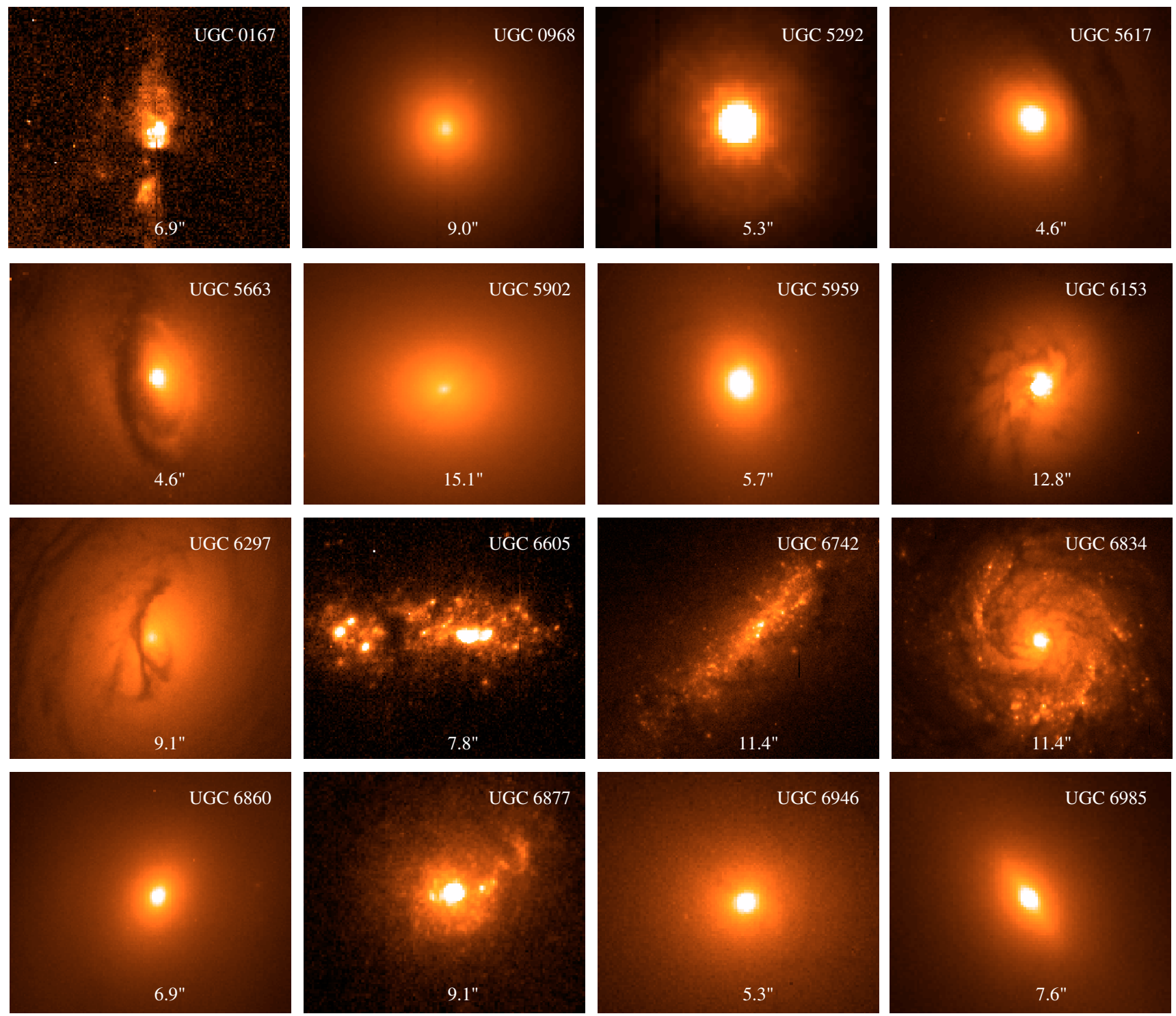

Fig. 1. HST images of the galaxies of the sample. The image size is shown in the bottom. The instrument/filter combination is reported in Tables 3 and 4. 
A. Capetti and B. Balmaverde: The host/AGN connection in nearby galaxies, Online Material p 3
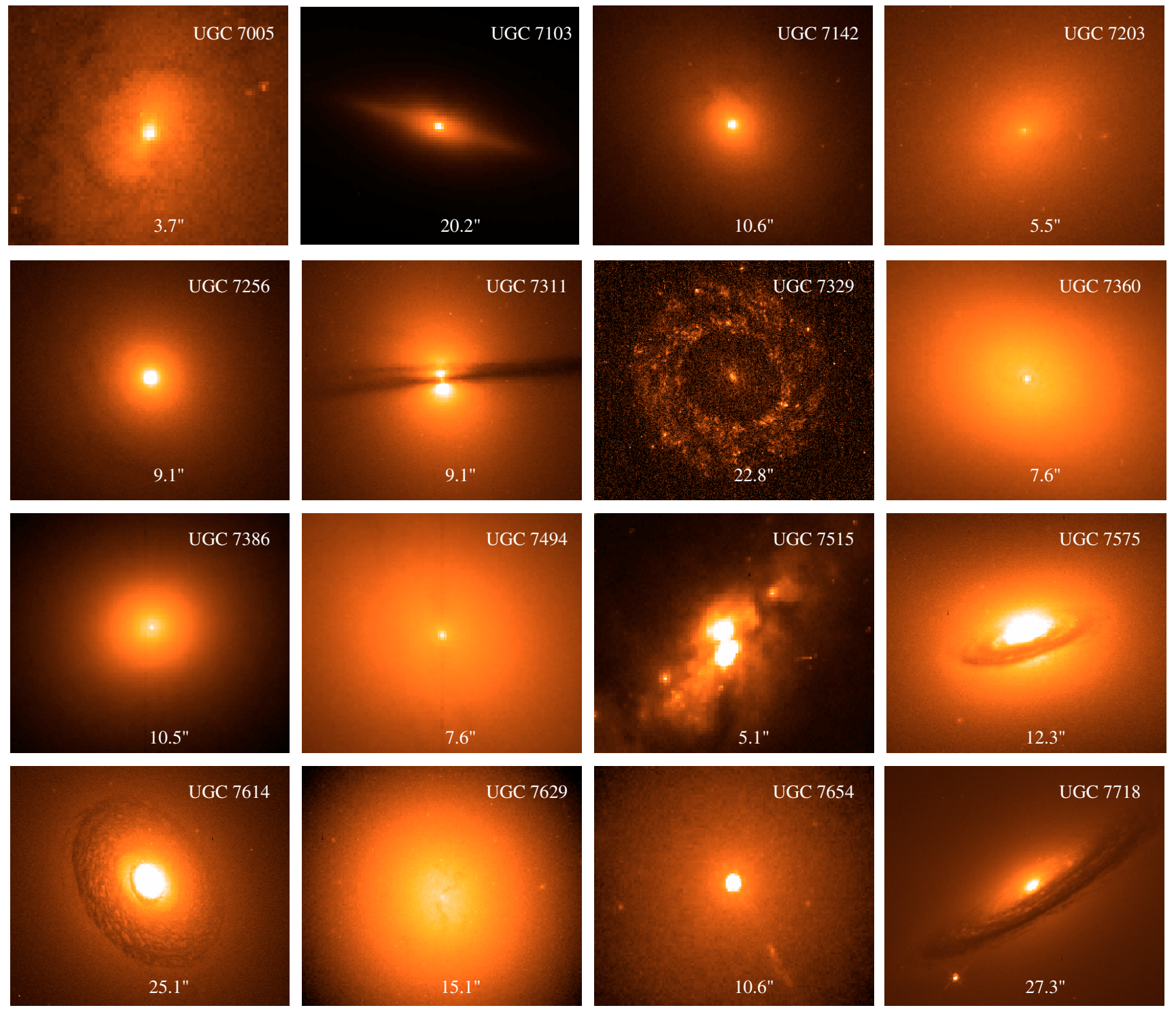

Fig. 2. HST images of the galaxies of the sample. 
A. Capetti and B. Balmaverde: The host/AGN connection in nearby galaxies, Online Material p 4
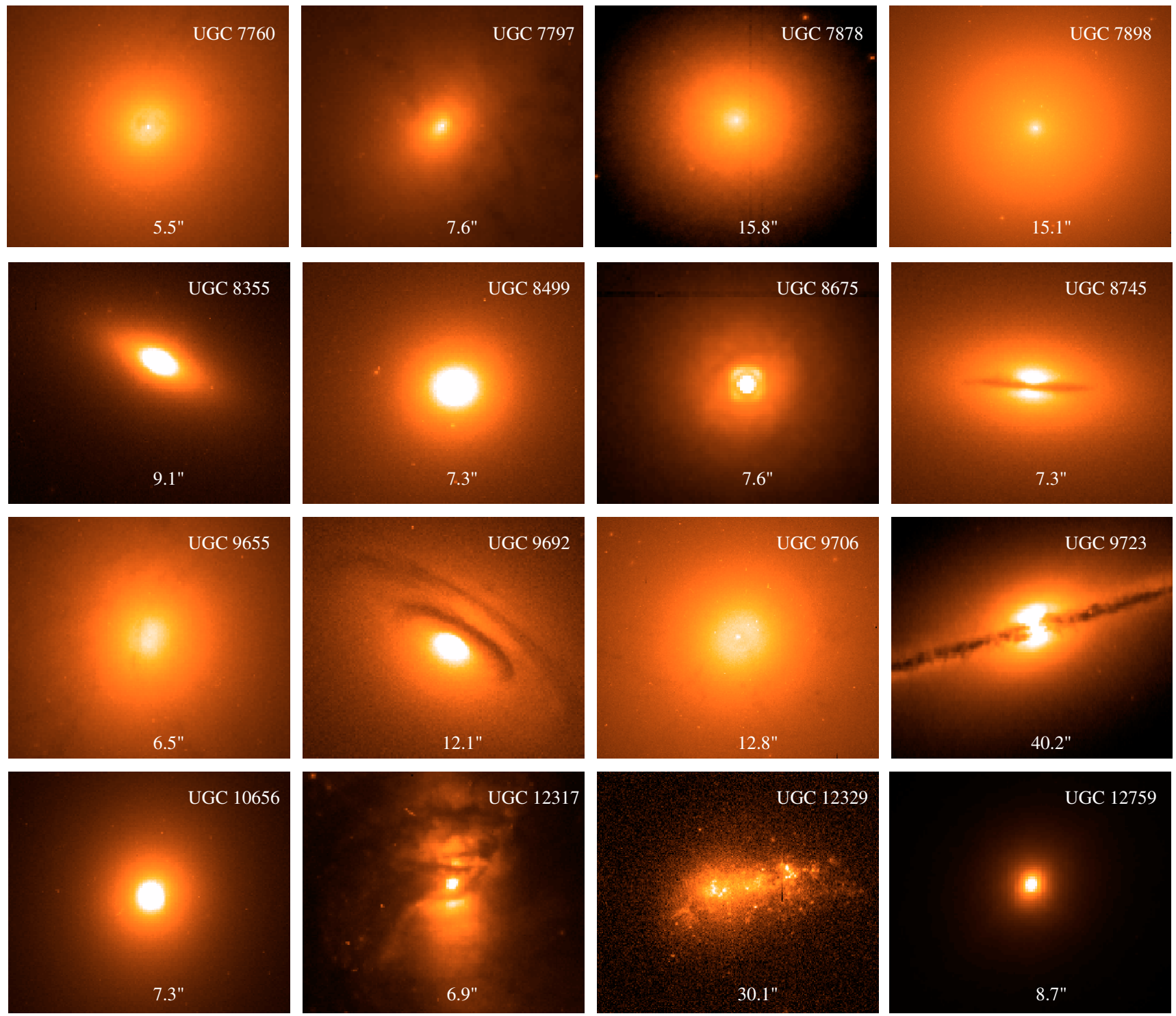

Fig. 3. HST images of the galaxies of the sample. 
A. Capetti and B. Balmaverde: The host/AGN connection in nearby galaxies, Online Material p 5
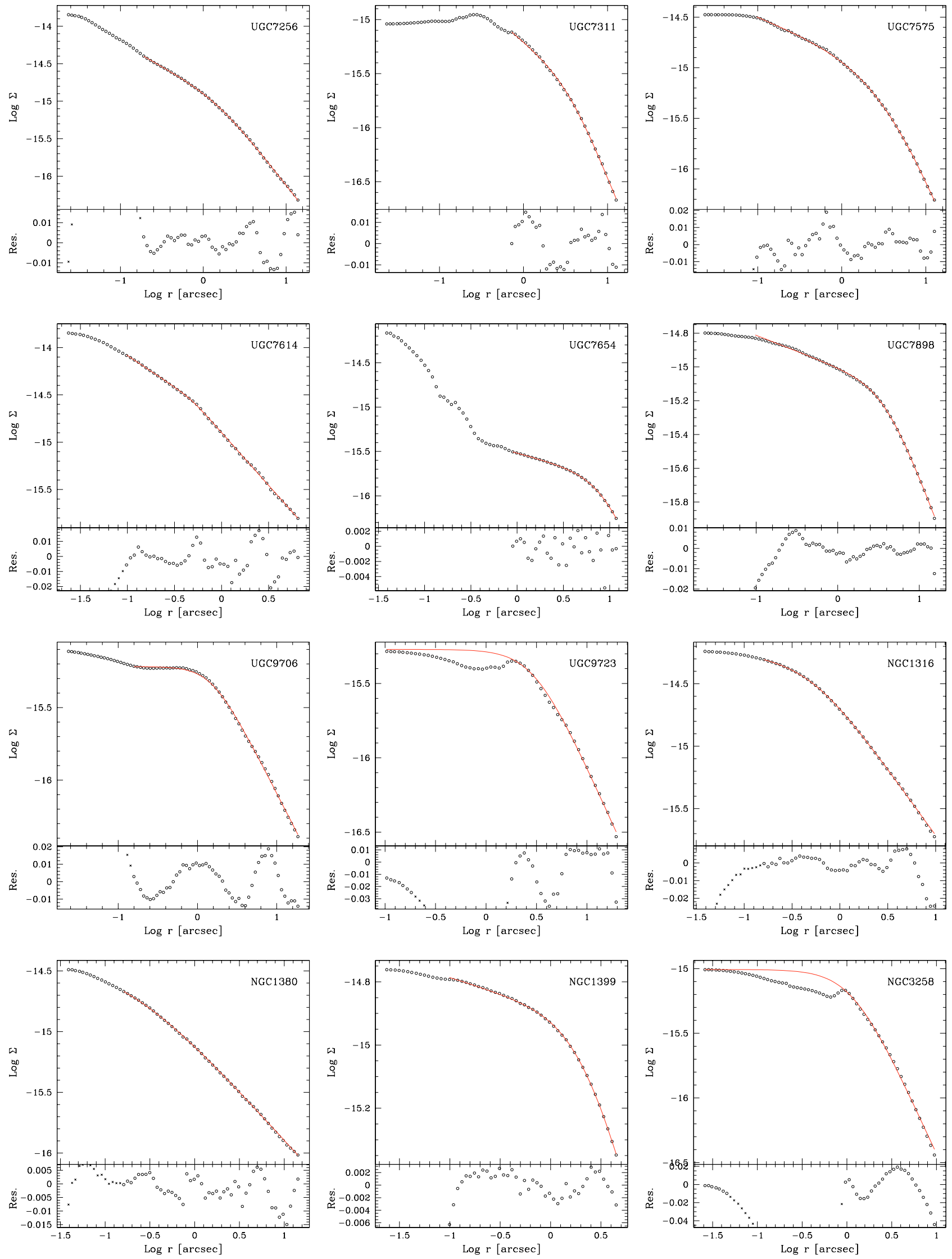

Fig. 6. Surface brightness (in unit of $\mathrm{erg} \mathrm{s}^{-1} \mathrm{~cm}^{-2} \AA^{-1}$ ) profiles of all galaxies lacking a core/power-law classification in the literature. 
A. Capetti and B. Balmaverde: The host/AGN connection in nearby galaxies, Online Material p 6
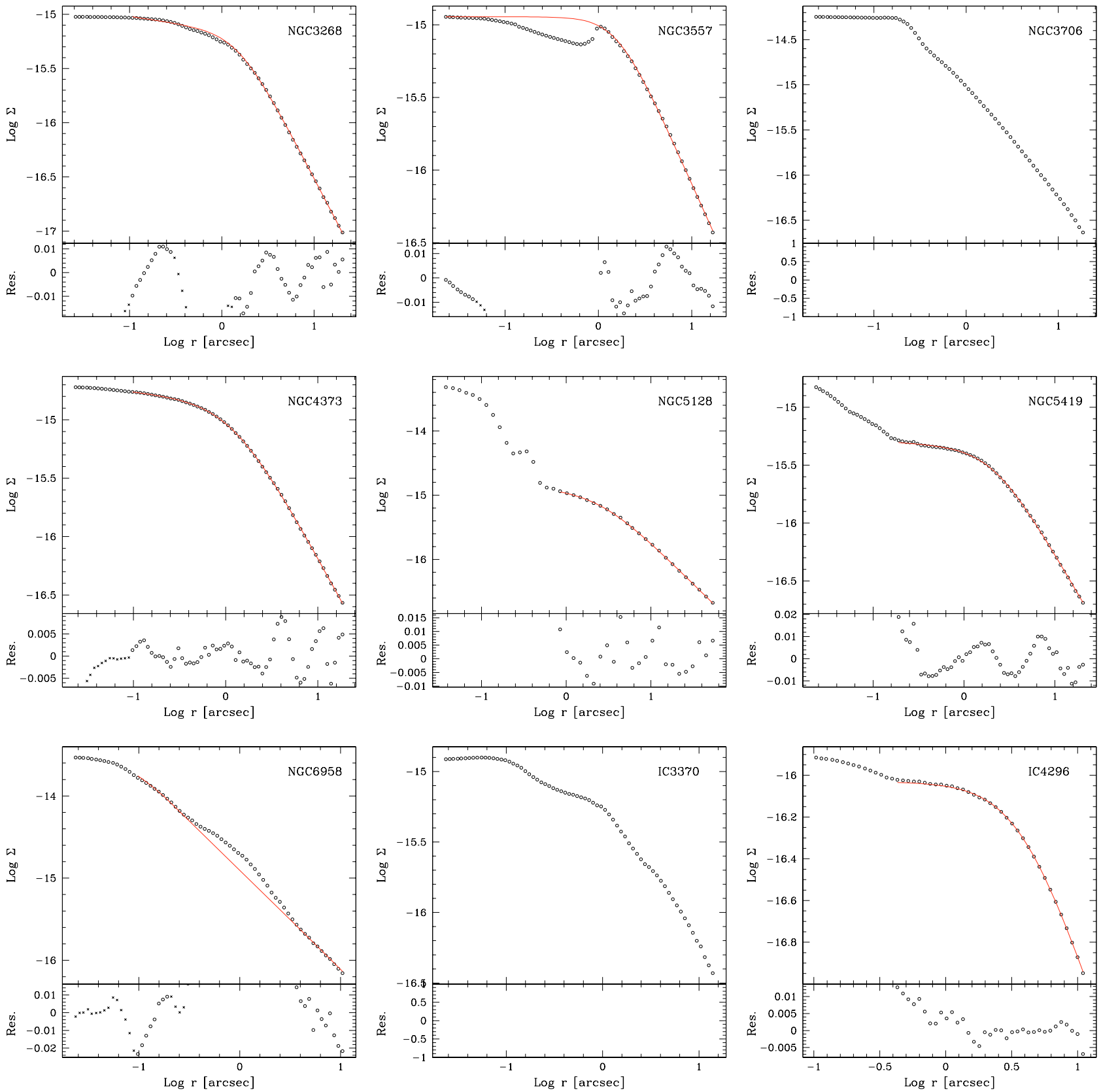

Fig. 7. Surface brightness (in unit of $\operatorname{erg~s}^{-1} \mathrm{~cm}^{-2} \AA^{-1}$ ) profiles of all galaxies lacking a core/power-law classification in the literature. 